\title{
Editorial
}

\section{Evidence-Dentistry in the XXI Century: Toward Clinically Relevant Complex Systematic Reviews (CRCSRs)}

\author{
Francesco Chiappelli ${ }^{1, *}$, Jaclyn Avila ${ }^{2}$, David Y. Chai ${ }^{2}$, Christine Dimaano ${ }^{2}$, Argina \\ Kudaverdian $^{2}$, Seong H. Park ${ }^{2}$, Sophia Peng ${ }^{2}$ and Sami Shahhal ${ }^{2}$ \\ ${ }^{I}$ Division of Oral Biology \& Medicine, UCLA School of Dentistry, USA \\ ${ }^{2}$ UCLA Pre-Dental Student Association, USA
}

At the onset, we must applaud the vision of the editorial board of The Open Dentistry Journal (TODENT J), and the publisher, Ms. Ajum Ara at Bentham Science, for realizing a special issue on evidence-based dentistry (EBD). It is a momentous occasion because it launches the second decade of the field: in fact, it was in 1999, that the American Dental Association (ADA) first made the pronouncement that EBD entailed the judicious integration of recommendations derived from the best available evidence, together with the dentist expertise, the patient's wants \& needs, laboratory tests and X-rays, and insurance coverage and other financial limitations, in order to reach a diagnosis, develop and treatment plan, and follow-up its outcomes.

Since then, it is appropriate to recall, the ADA has established a center for EBD under the able leadership of Dr. Julie Frantsve-Hawley, Director, and Dr. Krishna Aravamudhan, Assistant Director, Dr. Daniel M. Meyer, ADA Senior Vice President for Science \& Professional Affairs, and of course ADA President John S. Findley. The ADA EBD Center launched a most resourceful web-site (ebd.ada.org) earlier this year (3/10/09), which has met considerable success and dissemination.

The pursuance of the EBD model nationally and internationally has proven to be a tall order since, as noted by the 20092013 strategic plan developed a few months ago by the National Institute for Dental and Craniofacial Research (NIDCR), “...the oral cavity is an excellent model for studying complex systems. Home to a sophisticated set of functions, every second of every day the mouth exhibits a dynamic interplay between sensation, mechanical function and chemical flux. Multiple tissue types—including bone, nerves and blood_confront a bacterial zoo, crushing mechanical forces and electrical currents...." (NIDCR, Strategic Plan, 2009-2013). It is that very biological complexity that makes the field of oral biology and medicine a challenge for researchers and clinicians alike.

To achieve this goal, the ADA plays a critical role by training "Champions of EBD", who disseminate the principles of EBD among dental practices here and abroad. In addition, the ADA provides a service of Evidence Review, by which professionals, duly trained by the ADA EBD Center, review the published products of the scientific process of research synthesis, and present these summaries, both as part of the ADA EBD web site and the Journal of the American Dental Association (JADA) as properly processed and condensed, user-firendly reviews for utilization by clinical dentists.

These entreprises are in perfect consonance with the vision of clinical research and practice in dentistry for the next five years in the US, as articulated by Lawrence A. Tabak, D.D.S., Ph.D. Director, NIDCR: “...Scientific research holds great promise for targeted prevention and treatment strategies, and thus it is vital that oral health care become more science-based in the coming years ... to foster dissemination and implementation research to assure that research findings are translated into practice, policy and action in communities.... We can realistically improve our own oral health and that of our children and generations to come through creativity, diligence and adherence to a strong commitment to evidence-based and researchdriven practice and health outcomes....", as well as the overall vision of the pursuit of NIDCR ("...the NIDCR will seek to engage researchers in ... vital areas include(ing) ... the integration of evidence-based health recommendations into communities...”). Moreover, “...the NIDCR ensures that the American public has access to science-based, unbiased, accessible information that all individuals can use to make wise decisions about their oral health..." (NIDCR, Strategic Plan, 20092013). In brief, NIDCR and ADA are clearly joined in their unwavering support of EBD for the next several years - hence, one might say, a Golden Age for EBD is rising!

EBD, to be sure, is still in its infancy: over the past ten years, we have developed some of the fundamental tools and techniques of research synthesis that permit the systematic performance of evidence-based research (EBR), which then allows the revision of clinical practice guidelines ( $\mathrm{rCPG}$ 's), and their integration - at least in a preliminary fashion - in evidence-based practice (EBPr). It will be still a few years before the process will become refined sufficiently, and accepted widely to lead to evidence-based policies (EBPo) in stomatological care. Together these issues form a mosaic, a magical mosaic of sorts, that grows, as science grows, and that perfects itself as it leads us toward the Truth, as the real purpose of the scientific endeavor. The articles in this special issue of TODENT $\mathbf{J}$ each represent a carefully selected component of this special mosaic, as they address one or the other domain of EBD, and come together to represent one view of the state of affairs of EBD at this juncture. 
This special issue of TODENT $\mathbf{J}$ collects a number of seminal original research and review papers that converge to respond to the call to pave the way for the new decade in EBD. Three fundamental sections may group these reports, albeit such a structure may be too rigid. Specifically, and following the initial introductory paper, which is an encouraging statement of fact entitled "Evidence based dentistry in a developing economy - the Nigerian example", in which Dr. Akadiri and colleagues discuss the emergence of EBD in the context of the health care system in Nigeria, as one example of an emerging economy, Plutzer and collaborators highlight a critical problem in EBD, which refers to missing data, drop-outs and incomplete data sets in a superb re-examination of their work ("Dealing with Missing Outcomes: Lessons from a Randomized Trial of a Prenatal Intervention to Prevent Early Childhood Caries"). Dr. Bauer articulates in "Probability-Utility Model for Managing Evidencebased Central Database", one major area of concern for EBD in the next decade is the establishment of user-friendly databases, and the integration of information technology within the evidence-based decision-making process. In a further exploration of this particularly salient issue for the future of EBD, Giacomelli and Covani explore the process of data mining and its role in EBD in "Bioinformatics and data mining studies in oral genomics and proteomics: new trends and challenges". Dr. Bender emphasizes in "Using and Interpreting Adjusted NNT Measures in Biomedical Research", one fundamental component of the integration of research synthesis in the utilization of the best available evidence in clinical practice depends to a large extent on the number needed to treat (NNT) concept, which is explored in that article. A second section of this collection of papers, might be less research-directed and may be viewed to focus more of clinical issues. It includes the papers of Nocini and collaborators, in "Evidence-based dentistry in oral surgery: Could we do better?", bring to the surface the awareness that the field of EBD is not equally utilized across all dental specialties, such as particularly is the case in oral surgery. In a similar theme, Papadopoulos, in "Meta-analyses and Orthodontic Evidence-based Clinical Practice in the 21st Century", explores the role and integration of EBD specifically in orthodontics in the next decade. This awareness of the present limitation and need-to-grow of EBD as a field is further developed in the third section of this anthology, and include report by Kung and collaborators ("From Systematic Reviews to Clinical Recommendations for Evidence-Based Health Care: Validation of Revised Assessment of Multiple Systematic Reviews (R-AMSTAR) for Grading of Clinical Relevance"). Dr. Bauer and colleagues discuss the novel science of translational EBD, which holds strong promise in the next several years, in "Predictor Model of Root Caries in Older Adults: Reporting of evidence to The Translational Evidence Mechanism". In "The Translation Evidence Mechanism. The Compact between Researcher and Clinician", Dr. Bauer and Chiappelli discuss the intertwining of EBR and EBPr in the actualization of the EBD paradigm.

The articles presented in this issue could at best point to the future, but they cannot describe it. What must be done, however, is to look forward as we train the younger generation of EBD researchers and providers, and identify where work is imminently needed, where progress is urgently demanded, and where improvements to the field are most likely to occur in the short, the medium and the longer term. I have been very fortunate to work closely with a group of most promising pre-dental students while I was training them in EBD. They are listed as most deserving co-authors of this editorial because they made an invaluable contribution to one particular domain, which the field sees as the "next frontier" in EBD. That is to say, as systematic reviews accumulate on a given topic, the science of research synthesis will demand that they, somehow, be synthesized (e.g., systematic reviews traditionally incorporate primary research such as clinical trials and observational studies). The Kung paper touches on that very issue: what to do, and how to handle multiple systematic reviews on a given topic, particularly when they fail to propose similar consensus statements; that is to say, how to handle "discordant systematic reviews". Following the lead proposed by the ADA Center for EBD, this brilliant group of students excogitated an approach to synthesize multiple systematic reviews, and to coalesce them with primary research in a given domain, hence generating "complex systematic reviews" (aka, "meta-systematic reviews"). A better name perhaps might be Clinically Relevant Complex Systematic Reviews (CRCSRs): we shall see if this concept, name and approach weather the test of time. The details of the CRCSR process would be inappropriate to describe at this point, because it still needs to be perfected and presented to the scientific community; but, this ongoing work certainly gives a flavor for where the field is heading in the next few years.

Other important domains of EBD that are being explored, and that will become routine in the next few years include EBR on diagnostic studies, because EBD has largely been performed on prognostic clinical situations. It is important that we define how to obtain the best available evidence for establishing the presence or the absence of a condition (i.e., diagnosis), as well as for following the course of the disease and the success or failure of treatment (i.e., prognosis). Furthermore, it is increasingly recognized that the pursuit of the best available evidence in the EBD paradigm cannot, and must not rely only of the "level of the evidence" - meaning "what" was done: was it a clinical trial or an observational study; but must include as well the "quality of the evidence" - meaning "how" the study was conducted. Simply stated, a high level of evidence study (e.g., randomized clinical trial) that is performed poorly will generate evidence that could carry significant potential harm to the patient.

Research has established that, based on widely accepted standards of research methodology, design and data analysis, criteria can be developed that will accurately quantify the "quality of the evidence". Based on those criteria, sub-standard reports, what ever their "level of evidence", can be excluded by a process based upon statistical inference (i.e., acceptable sampling analysis). The remaining lot then represents the "best" of all of the "available" evidence. The Kung et al paper describes how this approach can be effectively utilized to rate systematic reviews, and to provide the clinician with userfriendly criteria to determine which consensus statement to use, or to avoid in the evidence-based clinical decision-making process.

It is self-evident that, if the field opens its horizons beyond the constraints of the "level of evidence", then several important hurdles will be resolved. For example, in several clinical fields, particularly in dentistry, clinical trials are close to impossible to 
perform, and observational studies are the best and often the only design possible. Looking at them from the viewpoint of the "level of the evidence", systematic reviews consistently decry a lack of evidence. This is a disservice to the profession. As the field increasingly considers the "quality of the evidence, then the value of these studies for the benefit of evidence-based dental practice will emerge, and our patients will be better served. Secondly, by looking at the "level of the evidence", one dismisses fundamental research (i.e., laboratory bench research, including materials research, animal studies); that produces a terrible drawback for the field and the profession, particularly in dentistry, because ours is a profession that relies on research aimed at characterizing new materials and fundamental biological principles to understand mechanisms in order to develop effective treatment interventions and, following pre-testing in animal models, to test experimentally with human samples. Therefore, if one looks strictly at the "level of evidence", this entire body of preparatory - one could say - dental research is considered to be of such low level to be close to useless.

Heavens no!, and to the rescue of this gross misrepresentation of the research needs and advances in our profession comes the view of the "quality of the evidence". In fact, criteria of good-bad methodology, design and data analysis can be applied equally to clinical trials, observational and diagnostic studies, cellular and molecular experiments, animal testing, and translational research.

The future, the next ten years of work in EBD, will see an expansion of our abilities to perform reliable research synthesis and systematic reviews of all types of biomedical research, based on the "quality of the evidence" and acceptable sampling, and ultimately for the benefit of the patient. Perhaps, TODENT J will produce another milestone special issue on EBD in 2019, and perhaps reports on topics such as those will take center stage. Time will tell, what is certain is that today, and thanks in part to the vision of TODENT $\mathrm{J}$ to produce this special EBD issue, the field of EBD is at the tipping point. The future of EBD promises to be exciting.

For the present, we cannot close these thoughts without sincere thanks to Professor Xenia Brant, DDS, Professor Antonio Mata, DDS, PhD, Oluwadayo Oluwadara, DDS, PhDCand, Nicolas Quach, DDS, Manisha Ramchandani, DDS, Professor Emeritus Paul Shapshak, PhD, and Professor Susan Sutherland DDS for serving as ad-hoc peer reviewers for the present issue.

Professor Francesco Chiappelli, Ph.D. Oral Biology \& Medicine, and Associated Clinical Specialties, UCLA School of Dentistry, CHS 63-090 Los Angeles, CA 90095-1668, USA \& Faculty sponsor UCLA Pre-Dental Student Association, ADA Champion Evidence-Based Dentistry, President International Forum for Evidence-Based Decisions \& Comparative Effectiveness Research

E-mail: fchiappelli@dentistry.ucla.edu

(C) Chiappelli et al.; Licensee Bentham Open.

This is an open access article licensed under the terms of the Creative Commons Attribution Non-Commercial License (http://creativecommons.org/ licenses/by-nc/3.0/) which permits unrestricted, non-commercial use, distribution and reproduction in any medium, provided the work is properly cited. 